\title{
Application of Bother in patient reported outcomes instruments across cultures
}

\author{
Mary C Gawlicki ${ }^{1}$, Shawn M McKown ${ }^{2}$, Matthew J Talbert ${ }^{3 *}$ and Barbara A Brandt ${ }^{2}$
}

\begin{abstract}
Background: The objective of this study was to determine the applicability of the term bother, as used in Patient Reported Outcomes (PRO) instruments that will be translated into foreign languages from English for the United States. Bother is versatile in English for the U.S., in that it can describe negative mental states and physical sensations, as well as social disturbances. Bother has many different meanings across cultures, due to this versatility. Alternatives for bother were explored for future PRO instrument development.

Methods: A PRO instrument used to evaluate the degree of bother resulting from psoriasis was analyzed. This disease can negatively impact patients physically, emotionally and socially. Translations of bother were analyzed to determine its meaning when translated into other languages. Cognitive debriefing was conducted on psoriasis patients with the instrument containing bother. Following cognitive debriefing, a questionnaire was distributed to linguists and cognitive debriefing subjects to collect definitions of bother in each target language, and detail any difficulty with translation.

To establish alternatives to bother and demonstrate the breakdown of concepts within bother, translations of the Dermatology Quality of Life Index (DLQI) were analyzed. This instrument was selected for its focus on psoriasis and use of terminology that lacks the ambiguity of bother.

Results: An analysis of back-translations revealed that bother yielded a back-translation that was conceptually different from the source $20 \%$ of the time (5/26). Analysis of alternative terminology found in the DLQI revealed much greater conceptual equivalence when translated into other languages.

Conclusion: When developing the wording of PRO instruments, the terminology chosen should be applicable across languages to allow for international pooling and comparison of data. While all linguists and subjects of cognitive debriefing understood bother to have a negative connotation, a large variety of definitions and synonyms provided across languages showed a lack of conceptual equivalence. Ambiguity of the term across cultures may result in variation in translation, impacting subsequent international data pooling. Analysis of alternatives revealed that measurement of unambiguous terminology produces the greatest conceptual equivalency across languages and cultures.
\end{abstract}

\section{Background}

Any report of the status of a patient's health condition that comes directly from the patient, without interpretation of the patient's response by a clinician or anyone else, has been termed a patient-reported outcome (PRO) [1]. Dependent upon the disease or condition of interest, a PRO may be the sole source of data from which drug efficacy can be measured, while in others it may provide

\footnotetext{
* Correspondence: mtalbert@corptransinc.com

${ }^{3}$ Linguistic Validation, Corporate Translations Inc, 2039 W. Wabansia Ave., Chicago, IL 60647, USA

Full list of author information is available at the end of the article
}

supplementary information on how the disease and its treatment impact patients' functioning and feeling. PROs are collected in clinical trials via standardized questionnaires designed to measure an explicit construct such as symptoms, activity limitations, health status, health-related quality of life (HRQOL). The use of PROs as clinical trial endpoints continues to be widespread, with over $45 \%$ of all New Molecular Entity (NME) or Biologics License Applications (BLA) submissions between 2006 and 2010 utilizing these instruments in some capacity $[2,3]$.

There are many challenges when PROs are included in multinational clinical trials [4]. One challenge is to

\section{Biomed Central}


ensure that terminology is translated and understood in the manner intended. In order to achieve cultural equivalence, PRO instruments must be translated using the appropriate methodology. When PRO instruments are utilized in a multinational clinical trial, they must undergo translation and linguistic validation to ensure conceptual equivalence and proper adaptation for the target language and country. This process requires translation by two independent translators and subsequent reconciliation to reach consensus, creating a "harmonized" translation. A third translator then "back-translates" the text back into English [5]. The back-translation is then reviewed to ensure conceptual equivalence between the translation and source text. Upon completion of this review, in-country cognitive debriefing interviews are carried out with local subjects located by a recruiter living in the target country. The purpose of these interviews is to test subject comprehension and readability of the translated text, and to make necessary revisions to ensure and maximize conceptual equivalence and cultural appropriateness. Failure to maintain conceptual equivalence calls the validity of collected data into question and impedes international data pooling [6].

Bother is commonly used in PRO instruments to measure a combination of patient satisfaction and discomfort with the disease and/or treatment. Although generally understood to have a negative connotation, bother can be used to describe physical, emotional and social states in U.S. English. This ambiguity results in a wide range of definitions and interpretations across many languages and cultures. Achievement of conceptual equivalence, therefore, becomes much more difficult, possibly threatening the data validity and pooling in multinational clinical trials [7]. The purpose of this research is to explore the meaning of bother when translated into different languages, and to determine the degree of difficulty in achieving conceptual equivalence. Following the analysis of bother when translated, acceptable alternatives were explored for use in PRO instruments.

\section{Review of current literature}

The findings of a previous observational study of bother were analyzed. Its purpose was to find contexts in which bother is used in US English and to form a foundational definition. 2000 statements that included the word bother were randomly selected from a website called Livejournal. com. This site contains many users' public diaries in the form of an online blog entry on a topic of the writer's choice. The results of this observational study revealed that bother was used to refer to negative emotional feelings, pain in a physical sense, and preoccupation in one's own thoughts [8].

In a study to measure erectile dysfunction due to benign prostatic hyperplasia, subjects were asked to assess their ability to obtain an erection and their satisfaction with their ejaculation [9]. Additionally, subjects were asked to assess the level of bother associated with their erectile ability and ejaculatory dysfunction. The bother assessment for both erectile and ejaculatory dysfunction did not seem to yield additional useful data for assessment of erectile dysfunction. It was also found that throughout the 24-week study, the bother score remained stagnant, while the erection and ejaculatory assessment changed throughout the course of the study.

In a study of lower urinary tract symptoms, a PRO instrument was developed which utilizes bother to assess severity of symptoms [10]. During the development of the PRO instrument, bother proved to be difficult to measure, due to its many "widespread interpretations." In order to create clarity for the respondent and to improve data validity and pooling, the PRO instrument was developed with bother appearing in association with specific lower urinary tract symptoms, such as patient's work productivity impact, mental health and sexual health. Although it was not clear whether data validity improved as a result, this alleviated the issue of conceptual ambiguity.

Solutions to ambiguous terminology, such as bother, have been observed in previous studies. A translatability assessment [11] was applied to distress, which was hypothesized to have different meanings in other languages and cultures [12]. Distress was used in the Hospital Anxiety and Depression Scale (HADS), a questionnaire used to evaluate a patient's views of cancer treatment taking place in Israel, therefore requiring that the instrument be translated into Hebrew and Arabic. Because distress is perceived differently between the two languages, the developers surveyed psychologists, social workers and stress researchers who were fluent in Hebrew and Arabic in an effort to create a definition of distress compatible with both languages. Respondents were asked to define distress in their own language. There was no consensus on a definition for distress among those surveyed. However, the solution was to create two distinct definitions of distress: stress from anxiety, and stress from depression. This resulted in two questions broken down into more specific measures: one concerning a patient's anxiety with regard to their cancer and treatment, and another concerning a patient's depression resulting from their cancer and treatment.

\section{Methods}

In the psoriasis PRO instrument analyzed as part of this study, patients were asked to what degree their symptoms bothered them. The name of the PRO questionnaire, its developer and owner must remain strictly confidential, per contractual agreement. Questionnaire data and key terms have been presented and analyzed as appropriate to 
honor this agreement. It should be noted that psoriasis was the focus for this study, as the disease may cause physical, emotional and social adverse affects. The PRO instrument went through linguistic validation - the process of forward and back-translation, followed by in-country cognitive debriefing. After completing the linguistic validation, cognitive debriefing data concerning the translation of bother were analyzed.

Translation and linguistic validation [13] of the psoriasis PRO instrument using bother within a Numeric Rating Scale (NRS) was carried out in 26 languages: Arabic (Israel), Bulgarian (Bulgaria), Czech (Czech Republic), Danish (Denmark), Dutch (Belgium), Dutch (Netherlands), English (Canada), English (Israel), English (UK), French (Belgium), French (Canada), German (Austria), German (Germany), Hebrew (Israel), Hungarian (Hungary), Italian (Italy), Japanese (Japan), Norwegian (Norway), Polish (Poland), Romanian (Romania), Russian (Israel), Russian (Russia), Spanish (Colombia), Spanish (Mexico), Spanish (Spain) and Spanish (US). Translatability and interpretation of bother were explored through analysis of linguistic validation data. Additionally, linguists and cognitive debriefing subjects were surveyed to determine difficulties in translating the instrument and in defining bother.

Although bother can be utilized across multiple parts of speech in English, as a noun, verb, or adjective, only the adjectival form is utilized in the observed PRO instrument ("How bothered are you...."). As a result, all observed translations, back-translations, and cognitive debriefing results refer exclusively to one consistent part of speech. In order to gather feedback on foreign-language use of bother across multiple parts of speech, the followup survey provided to linguists and cognitive debriefing subjects asked respondents to define and provide comment on the adjectival form found in the instrument and the term bother itself, which may function as either a noun or a verb. Upon review and comparison of the provided definitions and responses, it was determined that the reported meanings of bother showed no meaningful difference across observed parts of speech in non-English languages.

Following linguistic validation, all back-translations and cognitive debriefing data were analyzed, with a focus on the translation of bother. The dictionary definition of bother was compared to the dictionary definition of the English back-translation, and discrepancies were recorded. Skin was analyzed as a control term for comparison. After linguistic validation was completed, a voluntary questionnaire was sent out to all linguists and cognitive debriefing subjects. Linguists were asked to provide the term they selected for bother in their language, how they defined bother in their translation, and to elaborate on any translation difficulties. Subjects were asked how they defined bother and in what contexts it may be used in their language.
It is theorized that including more specific terminology which identifies the adverse effects of a disease individually will yield greater conceptual equivalency when translated. Examples pertaining to psoriasis were found within the Dermatology Quality of Life Index (DLQI) [14]. The follow list shows items found in the DLQI that avoid the use of bother.

- Over the last week, how itchy, sore, painful or stinging has your skin been?

- Over the last week, how embarrassed or self conscious have you been because of your skin?

- Over the last week, how much has your skin interfered with you going shopping or looking after your home or yard?

- Over the last week, how much has your skin influenced the clothes you wear?

- Over the last week, how much has your skin affected any social or leisure activities?

- Over the last week, has your skin prevented you from working or studying?

- Over the last week, how much has your skin created problems with your partner or any of your close friends or relatives?

- Over the last week, how much has your skin caused any sexual difficulties?

๑ Dermatology Life Quality Index. A Y Finlay, G K Khan, April 1992.

To test the hypothesis that more specific terminology will yield greater conceptual equivalency in translation, the linguistic validation results of the selected terminology were compared to those of bother. The example used to test this hypothesis will be the DLQI.

The subjects of cognitive debriefing were volunteers who self-reported their psoriasis and gave consent to participate in the interview. Confidentiality was protected as subject names were not collected nor linked to any of the data they provided. No medical data was collected. Subject contributions were solely for linguistic research and to ensure comprehension of the translated text. Additionally, the manner in which participant data was collected was determined to be ethically acceptable by the Institutional Review Board Services of Aurora, Ontario in Canada.

\section{Results}

\section{Back-Translation Analysis}

An analysis of all harmonized translations of the psoriasis instrument containing bother showed that the term was back-translated as something conceptually different from the source 19\% (5/26) of the time. Examples of nonequivalent back-translations were discomfort, embarrassment and anxiety. Words that appeared most frequently 
as back-translations of bother and their respective definitions are as follows:

- Trouble: To disturb mental calm and contentment

- Annoy: To disturb another in a way that displeases, troubles or slightly irritates

- Disturb: To interrupt quiet, rest, peace or order

- Discomfort: Absence of comfort or ease, uneasiness, hardship or mild pain [15]

With the exception of discomfort, all of the most frequent back-translations were conceptually similar to bother and therefore contain the same level of ambiguity. The most significant back-translation outlier for bother was anxiety. Anxiety was observed to be the least conceptually similar to bother, as it refers to nervousness or agitation. Anxiety was the selected Russian back-translated term for bother in both Russia and Israel.

\section{Cognitive Debriefing Analysis}

Following forward and back translation, the psoriasis instrument underwent cognitive debriefing interviews with five subjects per country. The exception was Japanese for Japan, where only four subjects were debriefed, as recruiters were unable to find a fifth volunteer with psoriasis. Subjects had been diagnosed with psoriasis and were diverse as to age, gender and level of education. The Table 1 displays a breakdown of the cognitive debriefing sample. Suggested replacements or deletions of the translated word for bother by the subjects are summarized in Table 2.

Several anomalous findings resulted from cognitive debriefing analysis. During cognitive debriefing, one German subject suggested removing bother without replacement, and noted the German word for bother ("stören") to be a "weak term". Similarly, a subject in the United Kingdom was uncertain as to whether bother was to be used in a mental or physical context. This is significant in a psoriasis $\mathrm{PRO}$, as the disease may cause physical pain resulting from scaling or abrasions on the skin, or mental anguish

Table 1 Summary of characteristics of those who took part in cognitive debriefing

\begin{tabular}{lll}
\hline $\mathbf{n}=\mathbf{1 2 9}$ & & \\
\hline Gender & & \\
Males: $\mathrm{n}=64$ & & \\
Females: $\mathrm{n}=65$ & Age (years) & Education (years) \\
& 47.2 & 13.2 \\
Average & 47 & 13 \\
Median & 14.9 & 3.2 \\
Standard deviation & $18-84$ & $6-24$ \\
Minimum - Maximum & &
\end{tabular}

due to the skin's unsightly appearance. In this case, due to the ambiguity of bother, it is not clear whether the severities of mental or physical symptoms of psoriasis are being measured.

Although bother was understood and paraphrased correctly by all subjects, the dominant issues raised were widespread discrepancies of interpretation between subjects. This variation in meaning is explored below.

\section{Analysis of linguist and subject questionnaire responses:}

All linguists were asked to provide the term they selected for bother in their language, and explain how the term is interpreted in their language and country. Additionally, linguists were asked to provide synonyms in English for their selected translation of bother and note whether they had difficulty achieving conceptual equivalence in their language. Not all linguists involved in the linguistic validation project are represented in the questionnaire results, as participation was voluntary. Table 3 presents a summary of the responses of those who participated.

It was observed that the definition of bother, when translated, varies among different languages and cultures, accounting for the variance in understanding among subjects. For example, the Dutch term for bother describes something that is constant or chronic, while the Norwegian term refers to something that is temporary. In another example, the terms selected in French for Canada and French for Belgium differed conceptually. The definitions provided by linguists were "to disrupt or make a change that is not pleasant" in French for Canada, and "to be troubled, annoyed or embarrassed" in French for Belgium. Overall, linguists reported issues with translating bother. Four out of a sample of 19 linguists attributed translation difficulties to the ambiguity of the term. Three out of this same sample of 19 linguists attributed translation difficulties to the fact that the target language has no direct translation for bother.

Subjects' questionnaire responses revealed that the majority of subjects within a single language-country pair had common interpretations of bother. For example, all Bulgarian for Bulgaria subjects defined bother as troubled and uneasy, while all French for Belgium subjects defined it as embarrassment. In some cases, the same language validated in multiple countries yielded similar interpretations. For example, all Dutch-speaking subjects in both the Netherlands and Belgium interpreted bother as a chronic hindrance and something that is always there. Additionally, a majority of German-speaking subjects from Germany and Austria interpreted bother as discomfort. On the contrary, there were several instances in which a common language yielded different results for each country. While all Spanish for Mexico subjects interpreted bother as a provocation of anger, a majority of Spanish for Colombia subjects interpreted bother as 
Table 2 Notable issues with bother found during debriefing

\begin{tabular}{|c|c|}
\hline Arabic (Israel) & 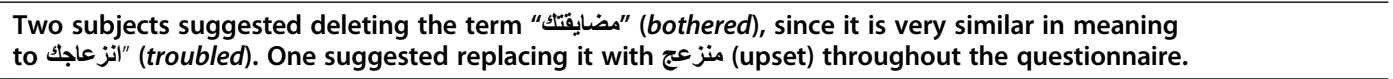 \\
\hline English (UK) & One subject was confused as to whether bother referred to physical or mental bother. \\
\hline French (Canada) & $\begin{array}{l}\text { One subject did not understand the word "l'importunité" (bothersomeness) and suggested using "le dérangement" } \\
\text { (bothersomeness) instead. }\end{array}$ \\
\hline German (Austria) & One subject noted that he makes no distinction in meaning between the words "störend" (bothered) and "lästig" (troubled). \\
\hline German (Germany) & $\begin{array}{l}\text { One subject suggested deleting "störend" (bothered) from one question.Two subjects would replace "störend" (bothered) } \\
\text { with "belastend" (troubled/stressed). }\end{array}$ \\
\hline Italian (Italy) & $\begin{array}{l}\text { One subject suggested replacing "misura del fastidio causato" (bothersomeness measure) with "valore del malessere dovuto" } \\
\text { (discomfort value) while another suggested replacing "fastidio" (bothersomeness) with "turbamento" (bothersomeness). } \\
\text { One subject suggested replacing "infastidiscono" (bothered) with "infastidisce" (bothered); another one suggested } \\
\text { replacing "infastidiscono" (bothered) with "disturbano" (disturbed). }\end{array}$ \\
\hline Spanish (Spain) & Two subjects suggested using only "molestias" (bothered) and not "preocupación" (troubled). \\
\hline
\end{tabular}

Table 3 Summary of linguist responses to the questionnaire

\begin{tabular}{|c|c|c|c|}
\hline Language (Country) & $\begin{array}{l}\text { Term selected in } \\
\text { target language }\end{array}$ & Definition of bother in target language & Difficulty with translation \\
\hline Bulgarian (Bulgaria) & безпокоя се & Troubled, annoyed, worried, inconvenienced. & $\begin{array}{l}\text { There are too many definitions of this concept in } \\
\text { Bulgarian. }\end{array}$ \\
\hline Czech (Czech Republic) & obtěžovat & $\begin{array}{l}\text { Something that makes one feel unpleasant or } \\
\text { uncomfortable. }\end{array}$ & None noted. \\
\hline Danish (Denmark) & Gene & Simply means something that bothers you. & None noted. \\
\hline Dutch (Belgium) & Last & $\begin{array}{l}\text { Annoyance or something that makes one feel } \\
\text { uncomfortable, or even causes pain. The term } \\
\text { can also describe something that is chronic. }\end{array}$ & None noted. \\
\hline French (Belgium) & Gene & $\begin{array}{l}\text { Being troubled, annoyed (could be physical), } \\
\text { embarrassed. }\end{array}$ & $\begin{array}{l}\text { Bother could mean "annoyance" or "worry" in } \\
\text { English and no French word. }\end{array}$ \\
\hline French (Canada) & Déranger & $\begin{array}{l}\text { Disrupt, introduce a change, or make something } \\
\text { different in way that is not pleasant. }\end{array}$ & $\begin{array}{l}\text { Translation was found to be very difficult because } \\
\text { the term is general in meaning. }\end{array}$ \\
\hline German (Austria) & Stören & Irritating or disturbing. & Difficult to find a German equivalent. \\
\hline German (Germany) & Störend & To be a nuisance. & $\begin{array}{l}\text { There are a wide variety of meanings and } \\
\text { associations for bother. }\end{array}$ \\
\hline Hebrew (Israel) & 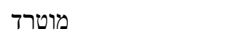 & To worry, be anxious or uneasy. & No difficulty translating. \\
\hline Hungarian (Hungary) & zavar & To disturb, distract or interfere with normal life. & Unable to determine an exact equivalent. \\
\hline Italian (Italy) & Fastidio & $\begin{array}{l}\text { The degree to which something may trouble or } \\
\text { the way one might dislike something. It may } \\
\text { also mean annoyed, irritated or aggravated. }\end{array}$ & $\begin{array}{l}\text { Was slightly difficult, but the selected term best } \\
\text { conveys the meaning. }\end{array}$ \\
\hline Japanese (Japan) & 悩む & Physical or psychological suffering. & $\begin{array}{l}\text { No, as Japanese is a vague language, so it was not } \\
\text { difficult to find another vague term. }\end{array}$ \\
\hline Norwegian (Norway) & Plage & $\begin{array}{l}\text { Someone or something that temporarily is } \\
\text { disturbing or troubling someone. }\end{array}$ & No issues translating. \\
\hline Polish (Poland) & Dokuczać & To be troublesome or a nuisance. & $\begin{array}{l}\text { No equivalent term in Polish to encompass all that } \\
\text { bother means in the context of mental and } \\
\text { physical issues. }\end{array}$ \\
\hline Romanian (Romania) & a deranja & $\begin{array}{l}\text { To cause discomfort, inconvenience, embarrass, } \\
\text { disrupt or trouble. }\end{array}$ & $\begin{array}{l}\text { No issues translating. Found the only acceptable } \\
\text { term. }\end{array}$ \\
\hline Russian (Israel) & беспокоить & To disturb, worry or trouble. & $\begin{array}{l}\text { No difficulties translating. Found the best term to } \\
\text { convey the intended meaning of bother. }\end{array}$ \\
\hline Russian (Russia) & Беспокоить & To cause anxiety, inconvenience, or trouble. & $\begin{array}{l}\text { Yes, because English has more synonyms than } \\
\text { Russian. }\end{array}$ \\
\hline Spanish (Mexico) & molestar & $\begin{array}{l}\text { It is a colloquial term used by doctors to ask } \\
\text { patients what bothers them. }\end{array}$ & $\begin{array}{l}\text { No. Selected term correlates with the meaning in } \\
\text { English. }\end{array}$ \\
\hline Spanish (Spain) & molestia & Sensation of discomfort or bother. & $\begin{array}{l}\text { Yes, as it was difficult to pick one of many possible } \\
\text { terms to convey the entire meaning of bother. }\end{array}$ \\
\hline
\end{tabular}


discomfort. Furthermore, all English for Canada subjects defined bother as difficulty or trouble, while all English for the United Kingdom subjects defined it as to irritate or to interrupt.

\section{Discussion}

A review of the four languages in this study for Israel (Hebrew, English, Arabic and Russian) revealed interesting results. As was the case with all subjects in the study, all Israeli subjects were asked to define bother as used in the target language for cognitive debriefing, as several of the subjects spoke a different native language, or were bilingual. Table 4 shows a breakdown of the native languages of each subject, in addition to other languages they speak fluently. English-speaking Israeli subjects did not conform to a common definition of bother. This could be attributed to the fact that English was not their native language, and regional differences in terminology. Definitions provided by a sample of four English-speaking Israeli subjects are as follows:

- To make anxious

- To cause inconvenience and disturbance

- To interfere and annoy

- To make problems

During cognitive debriefing interviews, subjects for Hebrew, Russian and Arabic for Israel reported a common interpretation of bother. On the contrary, linguists

Table 4 Languages of the Israeli subjects

\begin{tabular}{lll}
\hline Language & Native language & $\begin{array}{l}\text { Other languages known } \\
\text { by the subject }\end{array}$ \\
\hline Arabic (1) & Arabic & none \\
Arabic (2) & Arabic & none \\
Arabic (3) & Arabic & none \\
Arabic (4) & Arabic & none \\
Arabic (5) & Arabic & none \\
English (1) & Russian & Hebrew and English \\
English (2) & Russian & English, Hebrew and Ukrainian \\
English (3) & Russian & Hebrew, English, Arabic \\
English (4) & English & Hebrew \\
Hebrew (1) & Hebrew & None \\
Hebrew (2) & Serbian & Hebrew \\
Hebrew (3) & Hebrew & none \\
Hebrew (4) & Romanian & Hebrew \\
Hebrew (5) & Russian & Hebrew \\
Russian (1) & Russian & Hebrew \\
Russian (2) & Russian & Hebrew \\
Russian (3) & Russian & Hebrew \\
Russian (4) & Russian & Hebrew \\
\hline & & \\
\hline & &
\end{tabular}

for these languages did not share a common interpretation of bother, as demonstrated below:

- Hebrew - Negative connotation (that can be used in a number of contexts)

- Russian - Disturbance

- Arabic - Discomfort

Despite having a native tongue other than the questionnaire's target language, or bilingualism, a discrepancy in the interpretation of bother is still apparent in the results, indicating that a level of bother is a problematic measure when attempting to pool data across residents of Israel.

Terminology found in the DLQI analyzed as an alternative to bother revealed that more specific terminology will yield greater conceptual equivalency. The back-translated English words of the specific terms were compared to the source English words of the DLQI, mirroring the analysis used for bother. It was often observed that the specific terminology found in the DLQI back-translation was identical to the source. There were a few instances where the back-translated word was different from the English source, but still conceptually equivalent. Examples of such are interfered back-translated as prevented or disrupted, and self-conscious back-translated as ashamed. Table 5 compares the achievement of conceptual equivalence of specific alternative terminology as compared to bother. There were 22 available DLQI translations for analysis and 26 translations of the psoriasis instrument containing bother were available for analysis.

As shown in Table 5, the occurrence of achieving conceptual equivalence with translation of the more specific terminology was at least 95.5\% (21/22) in all cases. Examples

Table 5 Achievement of conceptual equivalence of DLQI concepts vs. bother

\begin{tabular}{ll}
\hline DLQI question concept & $\begin{array}{l}\text { Percentage of } \\
\text { back-translations } \\
\text { conceptually equivalent } \\
\text { to source } \mathbf{n}=\mathbf{2 2}\end{array}$ \\
\hline Itchy, sore, painful or stinging & $100 \%$ \\
Embarrassed or self conscious & $95.5 \%$ \\
Interfered & $100 \%$ \\
Influenced & $100 \%$ \\
Affected & $100 \%$ \\
Prevented & $95.5 \%$ \\
Creation of problems & $100 \%$ \\
Causing difficulty & $100 \%$ \\
\hline Bother question concept & $\mathbf{n = 2 6}$ \\
\hline $\begin{array}{l}\text { Bothered, bothersomeness (as related to } \\
\text { scaling and flakiness, skin thickening, } \\
\text { skin discoloration or redness) }\end{array}$ & $80 \%{ }^{*}$ \\
\hline *Different language/country pairs from DLOI. & \\
\hline
\end{tabular}


of back-translations that were not equivalent were selfconscious back-translated as shy and prevented backtranslated as caused a problem. Unlike bother, however, those issues were corrected easily after consultation with the linguists.

\section{Conclusion}

In development of PRO instruments, the terminology chosen should be applicable across languages to allow for multinational data pooling and comparison. The varied definitions of bother, as provided by linguists and subjects with psoriasis in response to the questionnaires, demonstrate that the term does not meet these criteria, and does not maintain conceptual equivalence across languages and countries when translated. Furthermore, as demonstrated by the findings within the four languages of Israel, bother may also present a lack of conceptual equivalence between languages within a single country. As further demonstrated by this research, the use of more specific concepts as an alternative of bother will yield greater conceptual equivalence across languages. To conclude, the use of bother in PRO instruments will threaten the validity of data and hinder data pooling from multinational studies. It is recommended that bother be avoided when developing PRO instruments intended for multinational clinical trials.

\section{Competing interests}

The authors declare that they have no competing interests.

\section{Authors' contributions}

MT conducted analyses of the linguistic validation results, conducted analyses of the questionnaire results, and drafted the manuscript. BB designed the study, created the questionnaires and made significant revisions to the first draft of the manuscript. All authors read, made revisions to and approved the final manuscript.

\section{Acknowledgements}

Ari Gnanasakthy for contributing

Staci White for editing and proofreading

Heather Cocco for desktop publishing.

Jason DeCoteau for contributing.

Alisa Heinzman for contributing.

\section{Author details}

'Corporate Translations Inc, 77 Hartland Street, East Hartford, CT 06108, USA ${ }^{2}$ Linguistic Validation, Corporate Translations Inc, 77 Hartland Street, East Hartford, CT 06108, USA. 'Linguistic Validation, Corporate Translations Inc, 2039 W. Wabansia Ave., Chicago, IL 60647, USA.

Received: 29 May 2013 Accepted: 30 January 2014 Published: 13 February 2014

\section{References}

1. US Food and Drug Administration Guidance for Industry: Patient-Reported Outcome Measures: Use in Medical Product Development to Support Labeling Claims. Rockville, MD: Department of Health and Human Services, Food and Drug Administration, Center for Drug Evaluation and Research; 2009.

2. Gnanasakthy A, Mordin M, Clark M, DeMuro C, Fehnel S, Copley-Merriman C: A review of patient-reported outcome labels in the United States: 2006 to 2010. Value in Health 2012, 15(3):437-442. May.
3. Hareendran A, Gnanasakthy A, Winnette R, Revicki D: Capturing patients' perspectives of treatment in clinical trials/drug development. Contemporary Clinical Trials 2012, 33(1):23-28.

4. Gnanasakthy A, DeMuro C, Boulton C: Integration of patient-reported outcomes in multiregional confirmatory clinical trials. Contemporary Clinical Trials 2013, 35(1):62-69.

5. Wild D, Eremenco S, Mear I, Martin M, Houchin C, Gawlicki M, et al: Multinational Trials-recommendations on the translations required, approaches to using the same language in different countries, and the approaches to support pooling the data: the ISPOR Patient-Reported Outcomes Translation and Linguistic Validation Good Research Practices Task Force Report. Value Health 2009, 12(4):430-440.

6. Wild D, Alyson G, Mona M, Sonya E, Sandra ME, Aneesa V-L, Pennifer E: "Principles of good practice for the translation and cultural adaptation process for Patient-Reported Outcomes (PRO) measures: report of the ISPOR task force for translation and cultural adaptation". Value Health 2005, 8:2.

7. Hareendran A, Williams AE, Tara S: "Pooling Data from Global Trials: Should There Be Specific Consideration for Pooling Data Collected Using Different Language Versions of a Patient-Reported Outcomes (PRO) Tool?"I ISPOR Connections 16.3. Lawrenceville, NJ: ISPOR; 2010.

8. Glynn D: Synonymy, lexical fields, and grammatical constructions: a study in usage-based Cognitive Semantics. In Cognitive Foundations of Linguistics Usage Patterns. Berlin: Mouton de Gruyter; 2010:89-118.

9. Hwang TIS, Chu S-H, Lin M-S, Chen C-S, Lee L-M, Chang H-C, Yeh S-D, Chen W-H, Chiang P-H: Impact of Alfuzosin on Sexual Function in Taiwanese Men with Benign Prostatic Hyperplasia. Kaohsiung J Med Sci 2012, 123:429-434.

10. Coyne KS, Sexton CC, Thompson CL, Milsom I, Irwin D, Kropp ZS, Kaplan S, Tubaro A, Aiyer LP, Wein AJ: The prevalence of Lower Urinary Tract Symptoms (LUTS) in the USA, the UK and Sweden: results from the Epidemiology of LUTS (EpiLUTS) Study. BJU Int 2009, 104:352-360.

11. Gawlicki M, Shawn MK: The Use of Face Validation to Create More Sound Translations." Presented by Corporate Translations. Prague, Czech Republic: Inc. at the International Society for Pharmacoeconomics and Outcomes Research; 2010.

12. Cohen M, Roni G, Tali C, Tamar S, Marian M, Abraham K: "Translating 'distress' and screening for emotional distress in multicultural cancer patients in Israel. Qual Life Res 2012, 21:555-562.

13. Gawlicki M: Procedures for Linguistic Validation of Health Status Questionnaires., CT: Corporate Translations, Inc.; 2004.

14. Finlay, Andrew $Y$ and Dr. Khan, G. K. The DLQI is copyright under the law of England and Wales. The DLQI is also copyright in the USA: The USA Library of Congress Registration Number: TXU 608406 Registration Date: 6 December 1993. The USA has reciprocal copyright agreements with most countries in the world thereby establishing worldwide copyright of the DLQI.๑ Dermatology Life Quality Index. A Y Finlay, G K Khan, April 1992 www.dermatology.org.uk. This must not be copied without the permission of the authors

15. "Trouble", "Annoy", "Disturb", \& "Discomfort". Oakland, CA: IAC Corporation; 2012. Dictionary.com

doi:10.1186/1477-7525-12-18

Cite this article as: Gawlicki et al:: Application of Bother in patient reported outcomes instruments across cultures. Health and Quality of Life Outcomes 2014 12:18. 